UNITED STATES DEPARTMENT OF THE INTERIOR

GEOLOGICAL SURVEY

LITHOLOGIC LOGS OF LISBURNE GROUP IN

LAWRENCE LIVERMORE LABORATORY DRILL HOLES 1 AND 2, CONFUSION CREEK, CHANDLER LAKE QUADRANGLE, NORTHERN ALASKA

By

William P. Brosgé and Augustus K. Armstrong

Open-file report $77-26$

1977

This report is preliminary and has not been edited or reviewed for conformity with Geological Survey standards 


\section{Lithologic logs of Lisburne group in Lawrence Livermore \\ Laboratory Drill Holes 1 and 2, Confusion Creek, Chandler Lake quadrangle, northern Alaska}

By William P. Brosgé and Augustus K. Armstrong

Two shallow test holes were drilled in the foothills of the Brooks Range by the Lawrence Livermore Laboratory, University of California in 1972 in order to make measurements of the electrical properties of permafrost. Cuttings and cores were given to the U.S. Geological Survey for examination, and the holes were later made available to the Survey for subsurface temperature measurements.

Location and history

The holes are located in sec. 26, T. 12 S., R. 1 W., at an elevation of about 2,700 feet on the crest of a low ridge about 1 mile west of Confusion Creek in the Siksikpuk River drainage (see fig. 1). Drill Hole 2 is about 550 feet east of Drill Hole 1.

The drilling contractor was Alaska Geological Consultants, Anchorage, Alaska. The two 6-inch holes were drilled with a DAMCO $2000 \mathrm{rig}$ and air hammer mounted on a Nodwell vehicle. They were completed in November, 1972, cased near the surface, and were capped after the electrical measurements had been made. In Drill Hole 2, and probably in Drill Hole 1, PVC tubing 1 inch in diameter was hung from the surface casing to the bottom of the hole, capped at the bottom, and left partly filled with oil. 
According to Lawrence Livermore Laboratory records, Drill Hole 1 (the west hole) hit fresh water at 592 feet and was drilled to a total depth of 600 feet. Nine days later a weak blow of gas was observed which continued for another 3 days. The nature of the gas was unknown, except that it would not burn and it extinguished matches. After the blow, 8 feet of water stood in the bottom of the hole.

Drill Hole 2 was drilled to a total depth of 570 feet. About 1 foot of fresh water stood in the bottom of the hole; no gas blow was noticed. Geologic setting and correlations

The drill holes are at the crest of a low west-plunging anticline that is probably the slightly offset westward prolongation of the much larger Tiglukpuk Creek anticline mapped by Patton and Tailleur (1964). Beds in and near the holes dip $10^{\circ}$ and less, so downhole measurements differ from true thickness of beds by less than 2 percent. The Lisburne Group crops out almost continuously along the crest of both anticlines, and the drill holes are entirely within the Alapah Limestone in the upper part of the Lisburne. A phosphatic zone at a depth of about 300 feet in each hole (fig. 2) can be correlated 1ithologically with the phosphatic black chert-shale member of the Alapah that is exposed in the section measured by H. N. Reiser on Tiglukpuk Creek about 5 miles east along strike (Patton and Tailleur, 1964, p. 420-423). The few microfossils found in the outcrops near the holes also indicate a correlation with the black chert-shale member and adjacent beds. These correlations suggest that a Mississippian supratidal environment was more persistent at the site of the drill holes than at the nearby exposed sections. If the correlation of the black chert-shale member is correct, many beds that are Iimestone at Tiglukpuk Creek are represented by dolomite at the drill site. 


\section{Microfossils}

Thin sections were made of cuttings from each of the numbered samples from Drill Hole 2 except sample number 46 . The foraminifera Earlandia sp., which ranges from Late Devonian to Early Permian, was found in two samples, number 9 (100 to 107 feet) and number 53 (490 to 495 feet). All other samples were barren.

Fifteen samples were also collected from the outcrops on the ridge crest east of Drill Hole 2, where the stratigraphic interval corresponding to the upper 200 to 300 feet of the hole is partly exposed. Brunsia sp., Archaediscus sp., Tetrataxis sp., and Endothyra? sp. were found in five samples near the top and bottom of this interval. This fauna probably represents the "Brunsia facies," which is also found in the Alapah Limestone section at Skimo Creek (fig. 1) and in the Alapah type section at Shainin Lake (Armstrong and others, 1970). In both those sections it occurs between the Upper Mississippian foraminiferal zones 13 and 16 , and at Shainin Lake it includes the black chert-shale member of the Alapah and adjacent beds.

The lack of microfossils from the suite of sections from Drill Hole 2 is clearly the result of environments of deposition and diagenetic events. The bulk of the thin sections are primarily lime mudstone (micrites) with little or no fossil bloclasts. They also are extensively dolomitized, and many of them have been dedolomitized. Even if microfossils had been present, these diagenetic changes would have destroyed them. Some thin sections contain bryozoan-echinoderm bioclasts and are wackestones and packstones. The rocks are extensively dolomitized. The dolomitization and silicification has occurred in the finer micritic materlal between the larger echinoderm and bryozoan fragments. Smaller microfossils such as foraminifera may have been destroyed by dolomitization. 


\section{Description of cuttings and cores}

The cuttings were sieved, examined with hand lens and tested with acid, and were described in the same terms of color and grain size as were used to describe the measured section on Tiglukpuk Creek. The logs in figure 2 were drawn from these descriptions. The cuttings in each numbered sample were uniform in rock type regardless of chip size, and therefore appeared not to be contaminated by cuttings from above. Insoluble residues of three samples were measured to determine the amount of argillaceous material in the limestones assigned to the black chert-shale member; no true shale was found. Phosphate was detected by testing with ammonium molybdate. The phosphate content of representative portions of samples 32 through 36 from Drill Hole 2 was measured quantitatively by S. T. Neil, U.S. Geological Survey, Menlo Park, California.

Thin sections of chips from each numbered sample in Drill Hole 2 were again described briefly during the examination for microfossils. In the following descriptive log the hand lens description of each sample is given first, followed by the thin section description in capital letters. 


\section{References cited}

Armstrong, A. K., Mamet, B. L., and Dutro, J. T., Jr., 1970, Foraminiferal zonation and carbonate facies of Carboniferous (Mississippian and Pennsylvanian) Lisburne group, central and eastern Brooks Range, arctic Alaska: Amer. Assoc. Petroleum Geologists Bull., v. 54, no. 5, p. 687-698.

Patton, W. W., Jr., and Tailleur, I. L., 1964, Geology of the KillikItkillik region, Alaska: U.S. Geol. Survey Prof. Paper 303-G, p. 409-500. 
Description of cuttings and cores

LLL Drill Hole 1 (west hole), Confusion Creek, 1972

Depth

in

Sample feet

$1 \quad 30-40$

$2 \quad 41-45$

$3 \quad 55-57$

$4 \quad 65-73$

$5 \quad 88-92$

$6 \quad 100-107$

$7 \quad 120-122$

$8 \quad 125-131$

$9 \quad 146-151$

$10 \quad 162-167$

$11 \quad 175-180$

CORE 187.75-

188.55

Dark gray fine-grained 1imestone; black chert

Dark gray fine-grained limestone

Same as above

Dark gray fine-grained limestone; medium gray mediumgrained limestone

Dark to medium gray fine-grained limestone

Light gray fine and coarse-crystalline dolomite and dolomitic limestone

Medium brown medium-grained limestone and dolomitic limestone

Dark gray fine-grained limestone

Medium brown fine-to medium-grained limestone; black chert

Medium brown fine-grained limestone; black chert

Dark brown fine-grained fetid limestone; black chert

Dark brownish gray medium-grained laminated bioclastic limestone; coral fragments; worm burrows; black chert.

Beds horizontal

12 ? $219-220$

Dark gray fine-grained thin-bedded limestone; black chert

$13 \quad 232-237$

$14 \quad 240-243$

Dark brown fine-grained limestone; brown argillaceous shaly limestone; black chert

Dark brown fine-grained limestone; black chert; pulverized rock is brown

$15 \quad 282-286$

Dark gray fine-grained platy limestone; black chert; pulverized rock is brown

$16 \quad 291-296 ?$

$17 \quad 296-298$

Same as above (Tag partly illegible)

Black fine-grained fetid shaly argillaceous limestone; a few clay shale partings; pulverized rock is dark brown

$18 \quad 302-305$

Same as above; a few chips are composed of $1 \mathrm{~mm}$ pellets of black phosphate rock

$19 \quad 320-330$

$20 \quad 346-350$

$21 \quad 351-362$

Dark brown fine-crystalline dolomite; 30 percent black chert

Medium brown medium-to fine-crystalline dolomite; black chert

Medium brown medium-crystalline dolomite

CORE 380.9-

381.25

Medium brown coarse-grained thick-bedded bioclastic limestone. Horizontal beds. Calcite-filled joints about 1 inch apart $\operatorname{dip} 60^{\circ}$

$22 \quad 427-435$

Light to medium brown fine- to medium-crystalline dolomite

$23 ? \quad 430-434$

$27 \quad 480-487$

Light brown medium-crystalline dolomite

Light brown fine-crystalline dolomite. Sample is almost all pulverized rock

$28 \quad 487-500$

Medium brown medium-grained limestone

$29 \quad 500-505$

Medium brown medium-grained limestpne; dark gray finecrystalline dolomite 


\section{Depth}

in

Sample feet

29 515-523 Medium brown medium-crystalline dolomite

30? 507-520 Light gray coarse-grained bioclastic limestone (Tag partly illegible)

31 ? -556 Medium brown medium-grained limestone; dark brown dolomite (Tag partly illegible)

32 562-567 Dark grayish brown fine- to medium-grained limestone

33 580-584 Dark gray fine- to medium-grained limestone

34 577-587 Carbonate and black chert. Sample is 90 percent gray dust

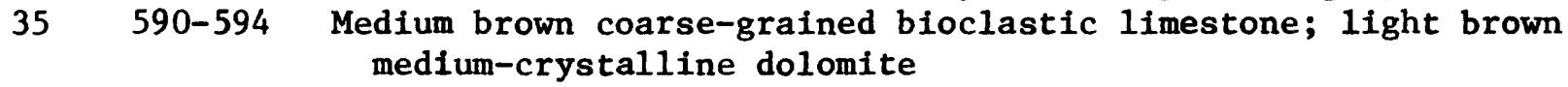

TOTAL DEPTH 600 FEET 
Description of cuttings and cores

LLL Drill Hole 2 (east hole), Confusion Creek, 1972

Depth

in

Sample feet

$111-14$

Dark gray fine-grained limestone. CALCITIC DOLOMITE AND CHERT; DOLOMTTE RHOMBS 50 MICRONS IN SIZE. RELIC STRUCTURE IN THE CHERT SUGGESTS THE ROCK WAS A PELLETOID LTME MUDSTONE.

$2 \quad 15-21$

Same as above. DOLOMITE, WITH 50 MICRON-SIZE RHOMBS, SOME DEDOLOMITIZATION AND INTERCRYSTALLINE CALCITE.

Same as above. SILICIFIED CALCITIC DOLOMITE AND CHERT.

$4 \quad 56-57$

$5 \quad 63-67$

Dark gray fine-grained shaly limestone; black chert; pulverized rock is black. ARGILLACEOUS CALCITIC DOLOMITE.

$6 \quad 84-87$

Medium gray and brown medium- to coarse-grained limestone; black chert. DOLOMITIC-SILICEOUS-BRYOZOAN-ECHINODERM WACKESTONE.

$7 \quad 90-95$

Light brown fine-grained dolomite and dark gray fine-grained limestone. PELLETOID-SILICEOUS-LIME MUDSTONE.

Dark to medium gray fine-grained limestone. SILICEOUSSPICULITIC-PELLETOID-BRYOZOAN WACKESTONE.

$8 \quad 95-99$

Dark gray medium-grained bioclastic 1imestone; black chert. BRYOZOAN-ECHINODERM PACKSTONE.

$9 \quad 100-107$

Medium gray calcilutite; white chert. BRYOZOAN-ECHINODERM WACKESTONES AND PACKSTONES.

$10 \quad 110-115$

$11 \quad 120-123$

Light to medium gray fine-grained platy, siliceous? limestone. DOLOMITIC AND NEOMORPHIC CALCITE--PROBABLY WAS LTME MUDSTONE.

Same as above; gray chert. ECHINODERM-WACKESTONE; VERY FINE FOSSIL FRAGMENTS AND NEOMORPHIC CALCITE CRYSTALS IN THE MICRITIC LTME MUD.

$12 \quad 126-128$

Dark gray fine-crystalline dolomite and silicified limestone; white chert. SPICULITIC CHERT AND DOLOMITE.

$13 \quad 128-130$

Light to dark gray very fine to fine-grained limestone. SILICEOUS LTME MUDSTONE.

$14 \quad 130-137$

Medium gray calcilutite; dark gray fine-grained limestone. SILICEOUS-PELLETOID-LIME MUDSTONE.

$15 \quad 150-156$

Medium gray fine-grained limestone. DOLOMITE; WITH 50 TO 100 MICRON-SIZE RHOMBS, EXTENSIVE DEDOLOMITIZATION.

$16 \quad 165-167$

Dark gray fine-grained argillaceous limestone; black chert; pulverized rock is black. DOLOMITE; IDIOTOPIC, WITH 50 MICRON-SIZE RHOMBS; DEDOLOMITE; CHERT; CLAY.

17 168-171 Gray limestone; white crystalline calcite; black chert. ECHINODERM-PELLETOID PACKSTONE; WELL SORTED.

$18 \quad 175-183$

Medium gray fine-grained limestone. CHERT AND SILICEOUSCALCITIC DOLOMITE.

$19 \quad 183-189$

Dark gray fine-grained limestone; calcite crystals. CHERT AND DOLOMITIC-LIME MUDSTONE.

$20 \quad 189-205$

Light gray fine-grained limestone; few chips; mostly pulverized rock. CHERT AND SILICEOUS DOLOMITE; DOLOMITE RHOMBS ARE 50 TO 150 MICRONS IN SIZE AND ARE HYPIDIOTOPIC AND COMMONLY IDIOTOPIC; CALCITE VEINS 


\begin{tabular}{|c|c|c|}
\hline Sample & $\begin{array}{l}\text { Depth } \\
\text { in } \\
\text { feet }\end{array}$ & \\
\hline 22 & $205-213$ & $\begin{array}{l}\text { Light gray coarse?-grained limestone; almost all pulverized. } \\
\text { NO THIN SECTION. }\end{array}$ \\
\hline 22 & $213-220$ & $\begin{array}{l}\text { Medium gray fine-grained dolomitic or silicified limestone. } \\
\text { NEOMORPHIC CALCITE, WITH RHOMBS } 20 \text { TO } 70 \text { MICRONS IN SIZE; } \\
\text { RECRYSTALLIZATION HAS DESTROYED ALL FORMER SEDIMENTARY FABRICS }\end{array}$ \\
\hline 23 & $219-227$ & $\begin{array}{l}\text { Medium dark gray fine-grained limestone. NEOMORPHIC CALCITE AS } \\
\text { ABOVE. }\end{array}$ \\
\hline 24 & $227-235$ & $\begin{array}{l}\text { Dark gray fine-grained shaly limestone. NEOMORPHIC CALCITE AS } \\
\text { ABOVE; BUT WITH WHAT APPEARS TO BE SILT-SIZE QUARTZ SAND } \\
\text { GRAINS IN THE } 30 \text { TO } 50 \text { MICRON-SIZE RANGE BETWEEN SOME OF THE } \\
\text { NEOMORPHIC CALCITE CRYSTALS. THE ROCK WAS PROBABLY ONCE AN } \\
\text { ARENACEOUS LIME MUDSTONE. }\end{array}$ \\
\hline 25 & $230-235$ & $\begin{array}{l}\text { Same as above. ARENACEOUS-BRACHIOPOD-ECHINODERM WACKESTONE TO } \\
\text { LIME MUDSTONE. THE MICRITIC FRACTION HAS UNDERGONE NEOMORPHIC } \\
\text { CRYSTAL GROWTH AS IN ABOVE SAMPLES. }\end{array}$ \\
\hline 25 & $235-240$ & $\begin{array}{l}\text { Same as above. IDIOTOPIC DOLOMITE RHOMBS IN CHERT AND } \\
\text { NEOMORPHIC-ARENACEOUS CALCITE. }\end{array}$ \\
\hline 26 & $240-245$ & $\begin{array}{l}\text { Same as above. Pulverized rock is dark gray. NEOMORPHIC } \\
\text { CALCITE, WITH } 30 \text { TO } 60 \text { MICRON RHOMBS--ROCK WAS PROBABLY ONCE } \\
\text { AN ARENACEOUS LIME MUDSTONE. }\end{array}$ \\
\hline 28 & $245-250$ & $\begin{array}{l}\text { Dark gray fine-grained limestone; black chert. DOLOMITIC- } \\
\text { NEOMORPHIC CALCITE. THIS THIN SECTION HAS EXTENSIVE EVIDENCE } \\
\text { OF DEDOLOMITIZATION, AND THAT MUCH OF THE CALCITE HAS } \\
\text { REPLACED DOLOMITE. }\end{array}$ \\
\hline 29 & $250-255$ & $\begin{array}{l}\text { Dark gray fine-grained thin-bedded limestone; black chert. } \\
\text { DOLOMITIC CALCITE; NEOMORPHIC; AS ABOVE. THIN SECTION IS AN } \\
\text { EXCELLENT EXAMPLE OF DEDOLOMITIZATION. }\end{array}$ \\
\hline 30 & $255-260$ & $\begin{array}{l}\text { Same as above; black chert abundant. NEOMORPHIC CALCITE; AS } \\
\text { ABOVE. }\end{array}$ \\
\hline 31 & $260-265$ & $\begin{array}{l}\text { Medium gray fine-grained thin-bedded limestone; black chert. } \\
\text { NEOMORPHIC CALCITE; AS ABOVE. }\end{array}$ \\
\hline
\end{tabular}

CORE 266

$32 \quad 268-275$

Dark gray fine-grained laminated limestone. Apparent dip $10^{\circ}$. Vertical and oblique calcite-filled joints 1 to 2 inches apart

$32 \quad 275-283$

Medium gray fine-grained limestone; black chert. LIME MUDSTONE.

Medium gray fine-grained thin-bedded dolomitic? limestone; black chert. 0.74 percent $\mathrm{P}_{2} \mathrm{O}_{5}$. DOLOMITIC-NEOMORPHIC CALCITE AS ABOVE.

33 283-287 Black fine-grained shaly argillaceous limestone consisting of about 80 percent $\mathrm{CaCO}_{3}$ and 20 percent insoluble clay and silt; black chert. 0.97 percent $\mathrm{P}_{2} \mathrm{O}_{5}$. ARGILLACEOUS-LIME MUDSTONE; VERY FINE-GRAINED WITH A FEW SHALL, CRINOID FRAGMENTS.

34 293-296 Same as above. About 1 percent of chips are composed of black phosphatic pellets about $1 \mathrm{~mm}$ in diameter. 5.92 percent $\mathrm{P}_{2} \mathrm{O}_{5}$. LIME MUDSTONE.

35 301-303 Dark gray fine-grained thin-bedded limestone consisting of about 95 percent $\mathrm{CaCO}_{3}$ and 5 percent insoluble material; black chert. 2.14 percent $\mathrm{P}_{2} \mathrm{O}_{5}$. NEOMORPHIC CALCITE; FORMED BY 50 TO 70 MICRON RHOMBIC PSEUDOMORPHS OF CALCITE AFTER DOLOMITE-A DEDOLOMITE. 


\begin{tabular}{|c|c|c|}
\hline ample & $\begin{array}{l}\text { Depth } \\
\text { in } \\
\text { feet }\end{array}$ & \\
\hline 36 & $305-310$ & $\begin{array}{l}\text { Dark brownish gray fine-grained shaly argillaceous limestone } \\
\text { consisting of about } 55 \text { percent } \mathrm{CaCO}_{3} \text { and } 45 \text { percent insoluble } \\
\text { particles; black chert. About } 1 \text { percent of chips are black } \\
\text { pelletal phosphate rock. } 9.41 \text { percent } \mathrm{P}_{2} \mathrm{O}_{5} \text {. SPICULITIC } \\
\text { ARGILLACEOUS LIME MUDSTONE. }\end{array}$ \\
\hline 37 & $330-335$ & $\begin{array}{l}\text { Medium gray medium-crystalline dolomite; black chert. DOLOMITE; } \\
\text { HYPIDIOTOPIC, WITH } 100 \text { TO } 200 \text { MICRON-SIZE RHOMBS. }\end{array}$ \\
\hline 38 & $335-348$ & $\begin{array}{l}\text { Medium brown medium-crystalline dolomite; black chert. DOLOMITE; } \\
\text { HYPIDIOTOPIC, WITH } 100 \text { TO } 300 \text { MICRON-SIZE RHOMBS; POOR SPACE } \\
\text { BETWEEN RHOMBS FILLED IN PART WITH SPARRY CALCITE. }\end{array}$ \\
\hline 40 & $352-355$ & $\begin{array}{l}\text { Light brown medium-crystalline dolomite; black fine-grained } \\
\text { thin-bedded limestone; black chert. CHERT AND HYPIDIOTOPIC } \\
\text { DOLOMITE. }\end{array}$ \\
\hline 40 & $357-361$ & $\begin{array}{l}\text { Light brown medium-crystalline dolomite. DOLOMITE; HYPIDIOTOPIC, } \\
\text { WITH } 100 \text { TO } 300 \text { MICRON-SIZE RHOMBS. }\end{array}$ \\
\hline 42 & $369-372$ & $\begin{array}{l}\text { Light brown coarse-grained platy dolomitic limestone; black chert. } \\
\text { ECHINODERM PACKSTONE; EXTENSIVE SECONDARY GRAIN GROWTH ON THE } \\
\text { LARGE ECHINODERM FRAGMENTS. }\end{array}$ \\
\hline 42 & $370-376$ & $\begin{array}{l}\text { Light brown coarse-grained platy dolomitic limestone; black chert; } \\
\text { minor white silicified dolomite. SILICEOUS-ECHINODERM-BRYOZOAN } \\
\text { PACKSTONE. }\end{array}$ \\
\hline 43 & $376-387$ & $\begin{array}{l}\text { Light brown coarse-grained dolomitic limestone; black chert. } \\
\text { ECHINODERM-BRYOZOAN PACKSTONE. }\end{array}$ \\
\hline $\begin{array}{l}44 \\
45\end{array}$ & $\begin{array}{l}387-397 \\
397-407\end{array}$ & $\begin{array}{l}\text { Light brown coarse-crystalline dolomite. CHERT AND LIME MUDSTONE. } \\
\text { Medium gray fine-crystalline dolomite. SILICEOUS AND RECRYSTAL- } \\
\text { LIZED LIME MUDSTONE AND PELLETOID-CRINOID WACKESTONE. }\end{array}$ \\
\hline 46 & $407-417$ & Medium brown fine-crystalline dolomite. NO THIN SECTION. \\
\hline 47 & $417-427$ & $\begin{array}{l}\text { Light brown fine-crystalline thin-bedded dolomite. CHERT AND } \\
\text { COARSE-GRAINED ECHINODERM PACKSTONE. }\end{array}$ \\
\hline 48 & $427-447$ & $\begin{array}{l}\text { Light brown coarse-grained bioclastic dolomitic limestone; black } \\
\text { chert. DOLOMITIC-COARSE-GRAINED-ECHINODERM PACKSTONE. }\end{array}$ \\
\hline 49 & $450-460$ & $\begin{array}{l}\text { Light to medium brown medium to coarse-crystalline dolomite. } \\
\text { DOLOMITE; HYPIDIOTOPIC, WITH } 300 \text { TO } 500 \text { MICRON-SIZE RHOMBS. }\end{array}$ \\
\hline $\begin{array}{l}50 \\
51\end{array}$ & $\begin{array}{l}465-470 \\
470-475\end{array}$ & $\begin{array}{l}\text { Same as above. LIME MUDSTONE AND DOLOMITE. } \\
\text { Light brown medium-crystalline dolomite; medium brown fine- } \\
\text { crystalline dolomite. CHERT AND DOLOMITE; HYPIDIOTOPIC, WITH } \\
100 \text { TO } 150 \text { MICRON-SIZE RHOMBS. }\end{array}$ \\
\hline 52 & $475-485$ & $\begin{array}{l}\text { Same as above; gray chert. CHERT AND DOLOMITIC COARSE-GRAINED } \\
\text { ECHINODERM PACKSTONE. }\end{array}$ \\
\hline 53 & $490-495$ & $\begin{array}{l}\text { Medium brown medium-crystalline dolomitic limestone. CHERT AND } \\
\text { COARSE-GRAINED HYPIDIOTOPIC DOLOMITE. }\end{array}$ \\
\hline 54 & $495-517$ & $\begin{array}{l}\text { Medium brown coarse-grained bloclastic limestone; medium brown } \\
\text { fine-crystalline dolomite. DOLOMITIC-ECHINODERM BRYOZOAN } \\
\text { PACKSTONE. }\end{array}$ \\
\hline 55 & $522-525$ & $\begin{array}{l}\text { Medium brown fine to medium-crystalline dolomite. DOLOMITE; } \\
\text { HYPIDIOTOPIC, WITH } 50 \text { TO } 100 \text { MICRON-SIZE RHOMBS. }\end{array}$ \\
\hline 56 & $525-531$ & $\begin{array}{l}\text { Same as above. DOLOMITE; HYPIDIOTOPIC, WITH } 150 \text { TO } 200 \text { MICRON- } \\
\text { SIZE RHOMBS. }\end{array}$ \\
\hline 57 & $532-533$ & $\begin{array}{l}\text { Medium gray medium-grained crinoidal limestone. CALCITE-DOLOMITE; } \\
\text { HYPIDIOTOPIC, WITH } 300 \text { TO } 500 \text { MICRON-SIZE RHOMBS. EXTENSIVELY } \\
\text { DEDOLOMITIZED. }\end{array}$ \\
\hline
\end{tabular}


$\begin{array}{cc} & \begin{array}{c}\text { Depth } \\ \text { in } \\ \text { Sample } \\ \text { feet }\end{array}\end{array}$

58 555-565 Medium gray fine-crystalline dolomite. SILICEOUS-CALCITICDOLOMITE; A VERY COMPLEX ROCK THAT HAS BEEN SILICIFIED IN PART, DOLOMITIZED, AND PARTLY DEDOLOMITIZED.

59 565-569 Light brown fine- to medium-grained limestone and dolomitic limestone. DOLOMITIC-ECHINODERM PACXKSTONE.

TOTAL DEPTH 570 FEET 


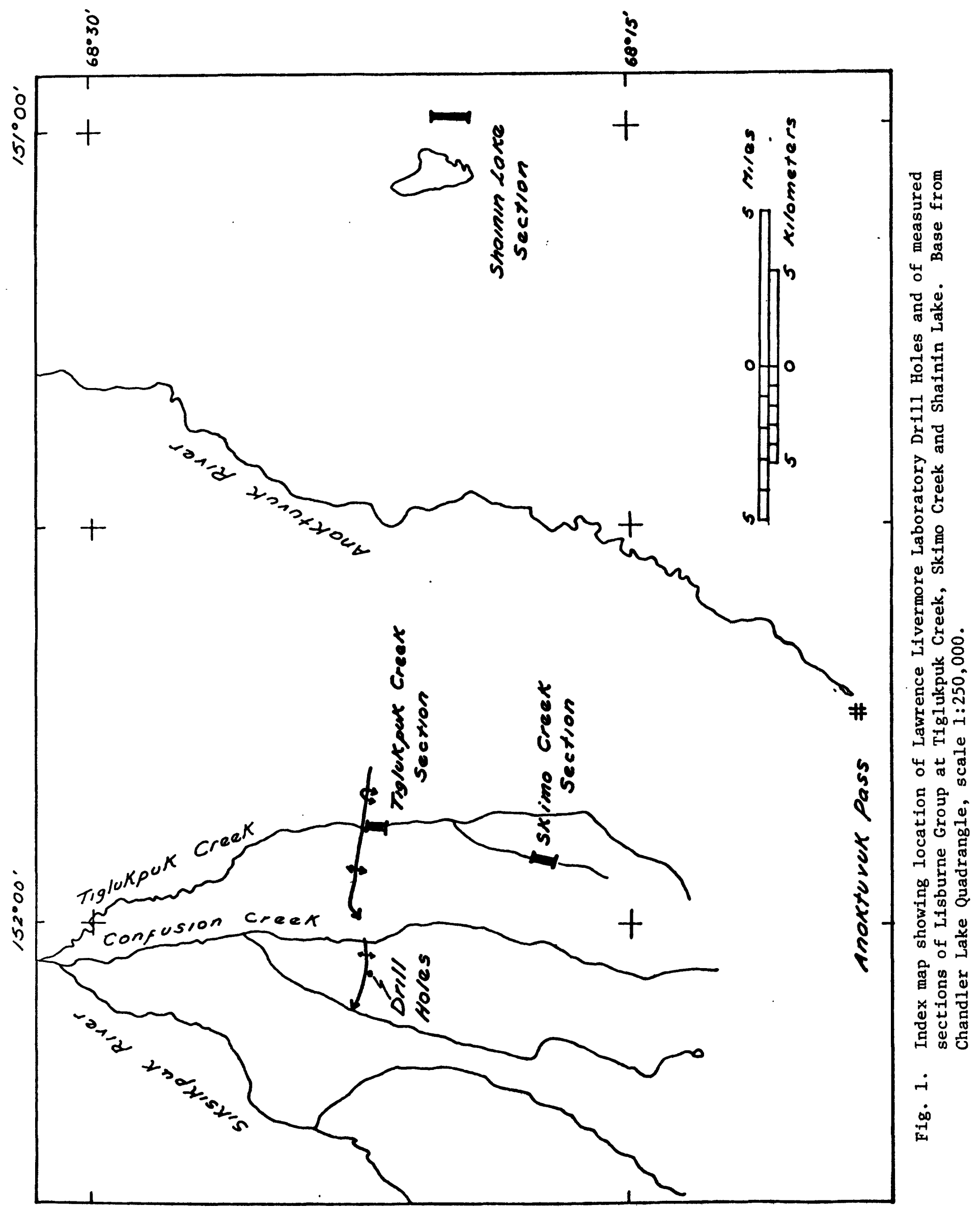

